\title{
Ultralight Structures for Space Solar Power Satellites
}

\author{
Manan Arya, Nicolas Lee†, and Sergio Pellegrino ${ }^{\ddagger}$ \\ California Institute of Technology, Pasadena, CA 91125
}

The design of a deployable spacecraft, measuring $60 \mathrm{~m} \times 60 \mathrm{~m}$, and with an areal density $100 \mathrm{~g} \mathrm{~m}^{-2}$, is described. This spacecraft can be packaged into a cylinder measuring $1.5 \mathrm{~m}$ in height and $1 \mathrm{~m}$ in diameter. It can be deployed to a flat configuration, where it acts as a stiff, lightweight support framework for multifunctional tiles that collect sunlight, generate electric power, and transmit to a ground station on Earth.

\section{Nomenclature}

\begin{tabular}{|c|c|c|c|}
\hline$B$ & Solar radiation pressure & $\alpha, \beta$ & Incident sunlight angles \\
\hline$E I$ & Beam bending stiffness & $\epsilon_{\max }$ & Maximum strain \\
\hline$f$ & Circle involute pitch & $\eta$ & Packaging efficiency \\
\hline$H_{p}$ & Container height & $\theta$ & Rotation \\
\hline$h$ & Flattened strip thickness & $\lambda$ & Linear density \\
\hline$i$ & Strip index & $\kappa$ & Signed curvature \\
\hline K & Stiffness matrix & $\sigma$ & Areal density \\
\hline$k$ & Number of strips in a quadrant & & \\
\hline$L$ & Spacecraft side length & & \\
\hline$l$ & Slip & & \\
\hline$M$ & Moment & & \\
\hline$m$ & Mass & & \\
\hline $\mathbf{n}$ & Curve normal & & \\
\hline $\mathbf{n}_{t}$ & Tile normal & & \\
\hline $\mathbf{p}, \mathbf{q}$ & Generator curves & & \\
\hline$p$ & Pitch & & \\
\hline $\mathbf{R}$ & Rotation matrix & & \\
\hline$R$ & Curve radius & & \\
\hline$R_{p}$ & Container radius & & \\
\hline $\mathbf{r}$ & Base curve & & \\
\hline$s$ & Arclength & & \\
\hline$T$ & Diagonal cord tension & & \\
\hline$V_{m}$ & Material volume & & \\
\hline$V_{p}$ & Container volume & & \\
\hline$w$ & Strip width & & \\
\hline$u$ & Vertical deflection & & \\
\hline
\end{tabular}

*Graduate Student, Graduate Aerospace Laboratories. MC 205-45. AIAA Student Member.

$\dagger^{\dagger}$ W. M. Keck Institute for Space Studies Postdoctoral Fellow in Aerospace. Currently, Engineering Research Associate at Stanford University, Stanford CA 94305. AIAA Member.

${ }^{\ddagger}$ Joyce and Kent Kresa Professor of Aeronautics and Professor of Civil Engineering, Graduate Aerospace Laboratories. MC 301-46. AIAA Fellow. 


\section{Introduction}

HEREIN is described a class of spacecraft composed primarily of a large number of independent multifunc-

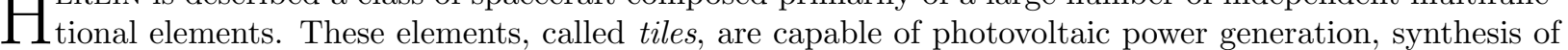
a microwave-frequency signal, and wireless power transmission. It is desired to fly a formation of many such spacecraft to create a space solar power system in geosynchronous orbit: a system to collect solar power and transmit it wirelessly to a ground station on Earth, as illustrated in Figure 1.

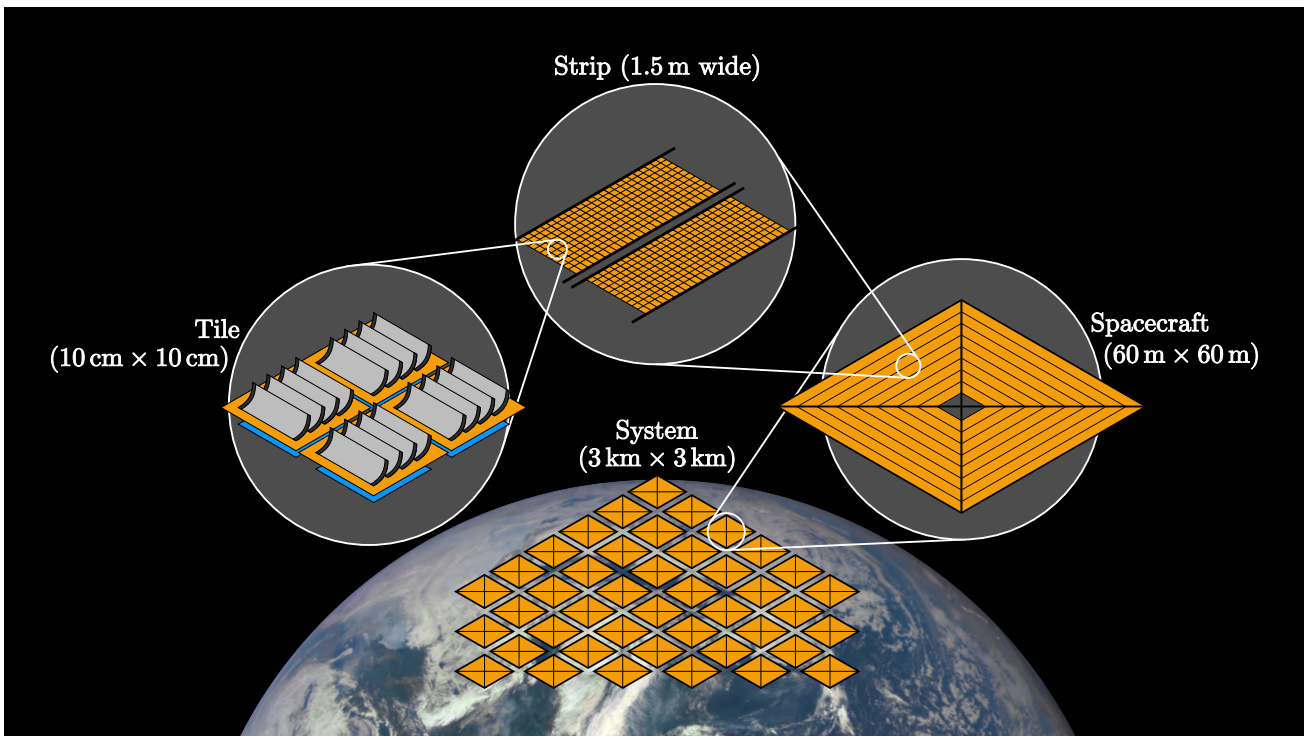

Figure 1: Overview of Space Solar Power System.

The tiles are interconnected and held in a planar configuration by a light, stiff structural framework, creating a spacecraft that measures $60 \mathrm{~m} \times 60 \mathrm{~m}$ and carries approximately 300,000 tiles. The geometry of this spacecraft is sheet-like: it has large in-plane dimensions (to collect solar power and to provide sufficient aperture for microwave power transmission), but comparatively small out-of-plane dimensions. For launch, the spacecraft can be packaged into a $\sim 1 \mathrm{~m}$ diameter, $1.5 \mathrm{~m}$ tall cylinder.

Compaction is enabled by the ability of each tile to be flattened, and to spring back into the original configuration when it is deployed. This flattening allows the spacecraft to be treated as a thin membrane, which is amenable to efficient methods of packaging. One particular design of a multifunctional tile capable of such flattening is briefly described.

This paper deals exclusively with the preliminary structural design of a single spacecraft. There are many aspects of the design, construction, and operation of such a space solar power system that are outside the scope of this paper (e.g. the design of lightweight photovoltaic cells, large-scale phased arrays across independent spacecraft, integrated circuits for microwave signal synthesis, and formation flying).

Similarly, there are many performance metrics that must be considered in the design of such a system (e.g. overall system power efficiency, total mass, capacity factor, specific power). However, the concern herein is purely structural design, and, as such, only relevant metrics will be considered. The key structural drivers are areal density, packaged volume, deployed stiffness, and deployment precision. Low areal density $\left(100 \mathrm{~g} \mathrm{~m}^{-2}\right)$ and low packaged volume are needed to reduce launch costs. Additionally, the spacecraft must be deployed to a precise shape, and maintain this shape within acceptable levels under applied loading. This paper will present a preliminary structural design of a spacecraft that has low areal density, small packaged volume, and is sufficiently stiff. The problem of precision deployment will be discussed in future work.

This paper is arranged thus. Section II provides background on relevant topics. Section III describes the design concept of the multifunctional tiles. Section IV presents the spacecraft structural architecture and the numerical model used to design this architecture. Section V discusses the spacecraft packaging concept. Section VI collects the results from these initial design exercises and presents a cohesive overview of the design of a single spacecraft. 


\section{Background}

This section provides a brief survey of three relevant bodies of work: the architecture and design of structures for space solar power systems, packaging schemes for membrane-like spacecraft structures, and the design of concentrating photovoltaic power systems for spacecraft.

\section{II.A. Structures for Space Solar Power Systems}

Early structural concepts for space solar power systems (see Figure $2 a$ ) tended to be massive and complex. ${ }^{1,2}$ These concepts comprise conventional structural systems (e.g. truss systems), designed to be assembled (by astronauts, robots, or a combination thereof) in orbit. The photovoltaic and power transmission systems are physically separate; the power transmission system is mounted on a gimbal that allows it to point to the ground station on Earth.

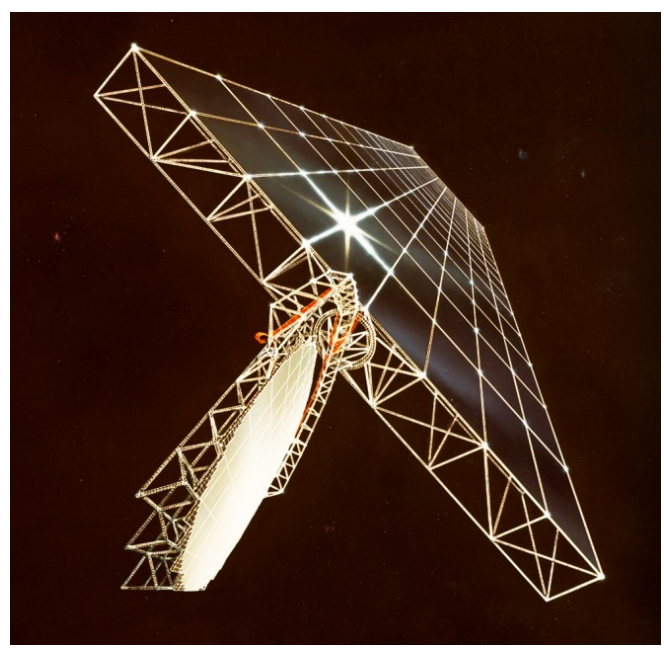

(a) NASA/DoE Reference Design ${ }^{2}$

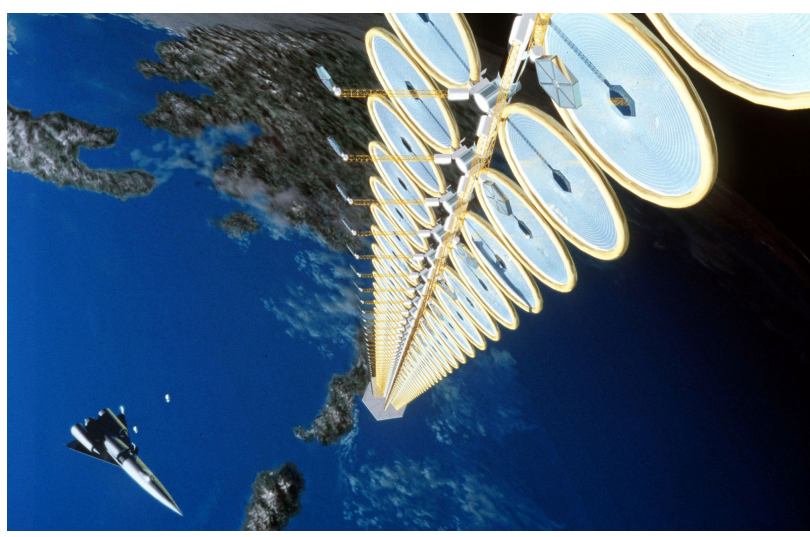

(c) NASA SunTower ${ }^{4}$

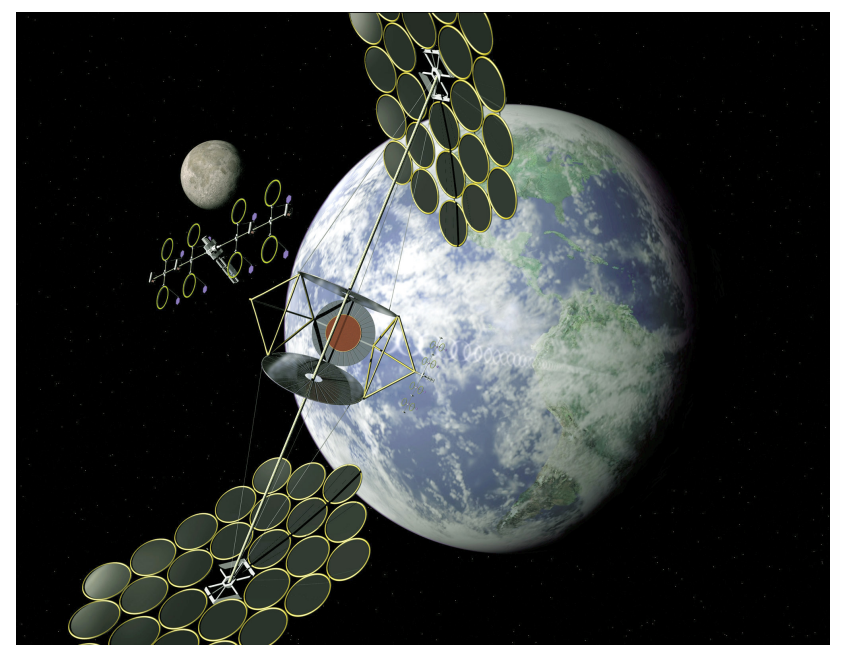

(b) Integrated Symmetrical Concentrator ${ }^{3}$

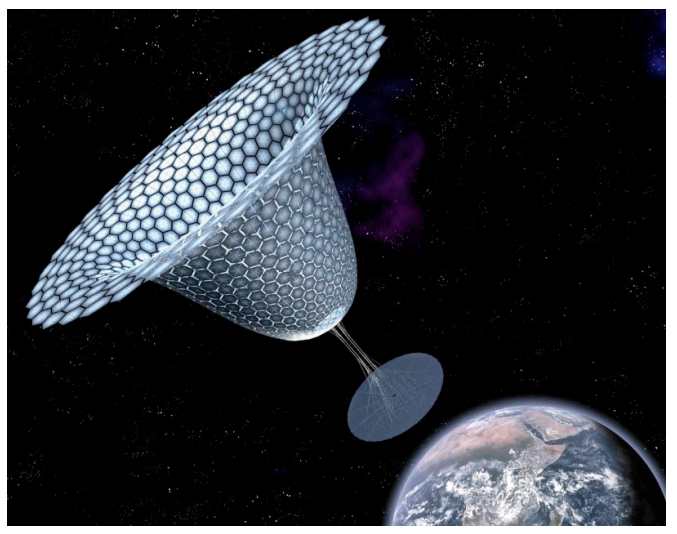

(d) SPS-ALPHA ${ }^{5}$

Figure 2: Space solar power system concepts.

Later space solar power concepts have driven towards modular and lightweight structures.

The Integrated Symmetrical Concentrator concept ${ }^{3}$ (see Figure 2b) consists of two reflector assemblies that direct sunlight to a central photovoltaic array, which is situated adjacent to the wireless power transmitter. The reflector assemblies can rotate about the axis of the system to direct sunlight onto the photovoltaic array. A later JAXA concept ${ }^{6}$ envisions the reflectors and the power-generation-and-transmission segment as separate spacecraft, flying in formation. This reduces structural complexity by removing the gimbal system.

The NASA SunTower concept ${ }^{4}$ (see Figure 2c) employs a gravity-gradient-stabilized structure that consists of a long central spine with many power-generating units arranged along its length. Each power- 
generating unit consists of a thin-film Fresnel concentrator (deployed and stabilized by an inflatable ring on the edge) and a photovoltaic unit. At the end of the spine is a wireless power transmission unit. The ESA SailTower concept ${ }^{7}$ is similar in architecture; however, the concentrating photovoltaics are replaced by thin-film photovoltaic blankets.

The SPS-ALPHA concept ${ }^{5}$ (see Figure $2 \mathrm{~d}$ ) is modular and robotically assembled in space. It comprises a large reflector system, a power-generation-and-transmission segment, and a connecting truss structure. The reflector system directs sunlight onto the power-generation-and-transmission segment, which converts it to electricity, and beams it to Earth. The reflector system is composed of many individual membrane reflective surfaces (akin to solar sails) that can be individually pointed.

The tethered JAXA concept ${ }^{8}$ consists of a single square plate-like structure that has photovoltaic elements on one side, and a phased array of antennas on the other. The structure is stabilized by gravity gradient forces; four tethers from the corners of the square plate are connected to a satellite bus that acts as a counterweight. The phased array can electronically steer the microwave beam, precluding the need for a gimbal system.

\section{II.B. Packaging of Membrane Structures}

Packaging techniques for membrane structures for space applications have been studied extensively. The reader is referred to an earlier paper ${ }^{9}$ for a fuller discussion of such techniques. Here, the discussion focuses on two ideas that are particularly relevant to the present work.

The IKAROS solar sail (a $7.5 \mu \mathrm{m}$ thick, $14 \mathrm{~m} \times 14 \mathrm{~m}$ square membrane) was packaged by folding and then wrapping. ${ }^{10}$ The four trapezoidal quadrants were individually z-folded, then connected together along the diagonals and wrapped around a cylindrical hub $1.6 \mathrm{~m}$ in diameter. If the sail had been constructed from a single sheet, the equivalent fold pattern would have consisted of a set of equally spaced concentric squares, alternating between mountain and valley folds, with additional folds connecting the corners of these squares. The packaging scheme used herein is very similar to that of IKAROS; however, instead of creases, slipping folds are used, which allow for the packaging of much thicker structures.

Slipping folds were introduced by the present authors in a previous paper, ${ }^{9}$ and they allow for both rotation about and translation along the fold line. In this previous paper, a set of parallel slipping folds were used to package square membranes very tightly, without extension, and without plastic deformation. They are crucial to the present packaging scheme.

\section{II.C. Concentrating Photovoltaic Systems for Spacecraft}

Using concentrating photovoltaic systems in space applications is attractive because of the potential for mass and cost savings. Concentration reduces the photovoltaic cell area, replacing that area with lightweight optical concentration elements. Here, a brief survey of such systems is provided.

The SCARLET solar array on the Deep Space 1 mission $^{11}$ uses an array of linear concentrators to illuminate photovoltaic cells. The concentrators are arched Fresnel lenses, $1 \mathrm{~cm}$ wide and $4 \mathrm{~cm}$ long. They operate at a concentration factor of 8 . The lenses are molded silicone with glass substrates, and are supported by graphite/epoxy frames. These frames stow against the plane of the photovoltaic cells, and are deployed to the functional focal length by lenticular tape springs. ${ }^{12}$

The Stretched Lens Solar Array ${ }^{13}$ has a similar optical configuration, but uses lighter flexible Fresnel lenses made from silicone rubber, without the glass substrate. These lenses can be flattened against the photovoltaic cells, and pop back up using spring-loaded arches at the ends of the lenses. Additionally, the Stretched Lens Solar Array uses thin composite material to support the photovoltaic cells, as compared to the honeycomb panel used in the SCARLET array. ${ }^{14}$

The FAST solar concentrator array ${ }^{15}$ uses reflective linear concentrators to focus light onto photovoltaic cells on the backside of the adjacent linear concentrator. These concentrators operate at a concentration factor of 12.5. Construction and deployment details for the FAST array could not be found in the published literature. 


\section{Multifunctional Tile Concept}

This section discusses the concept for a single tile capable of power generation and transmission, and describes the construction of an initial tile mockup. Of particular importance to the overall spacecraft packaging scheme is the ability of each tile to flatten, which is also discussed.

A tile measures $10 \mathrm{~cm} \times 10 \mathrm{~cm}$ in plane. The out-of-plane deployed dimension is as yet undecided, but is expected to be around $3 \mathrm{~cm}$. Each tile has five half-parabolic linear trough concentrators that focus light onto thin-film photovoltaic cells attached to the edge of the adjacent concentrator. This optical configuration is similar to that of the FAST array, ${ }^{15}$ though the tiles operate at a higher concentration factor, and are capable of flattening. The concentrators focus light onto a narrow photovoltaic cell.

These concentrators are supported by a ground layer. The ground layer provides an attachment point for the rest of the tile components, houses the antenna ground plane, and is used for routing generated power and signals. It also holds an integrated circuit that synthesizes a microwave signal, and amplifies it using generated power. Below the ground layer is an antenna, through which the amplified signal is transmitted.

The tile can flatten for packaging. As shown in Figure 3, the concentrator consists of a thin, aluminized polymer film supported at either end by carbon fiber springs. The concentrator edge springs have the appropriate parabolic profile needed for concentration. At the top edge, there is a thin carbon fiber rod attached to the concentrator that maintains straightness. Each concentrator is able to elastically flatten; the edge springs deform from their unstressed parabolic shape to flat. The patch antenna is held below the ground layer by four carbon fiber springs that have an "S" profile, and these springs can flatten such that the patch antenna plane rests directly below the ground layer. Figure 3 shows an initial mechanical mockup.

These tiles are functionally independent, and require no bulky power interconnections. Power generated by a tile is transmitted by the same tile. Only data and timing signals need to be exchanged between tiles.

Initial tile mockups, shown in Figure 3, were constructed thus: the ground layer was constructed using $7.5 \mu \mathrm{m}$-thick polyimide film (Dupont Kapton HN film), supported at the edges by a frame of $120 \mu \mathrm{m}$-thick pultruded carbon fiber rods. The patch antenna layer was built using a similar technique, using 7.5 um-thick polyimide film and a pultruded carbon fiber rod frame. The ground and antenna layers have representative conductive aluminum layers deposited on them. The "S" springs were constructed using carbon fiber composite material.

The concentrators were made using aluminized $25 \mu \mathrm{m}$-thick polyethylene film (Mylar), supported at the edges by carbon fiber composite springs. A pultruded carbon fiber rod was attached to the front surface, along the top edge of the concentrator, and a strip of photovoltaic material was attached to the back surface, along the top edge. The tile mockup can flatten and then elastically pop back into its operational state.

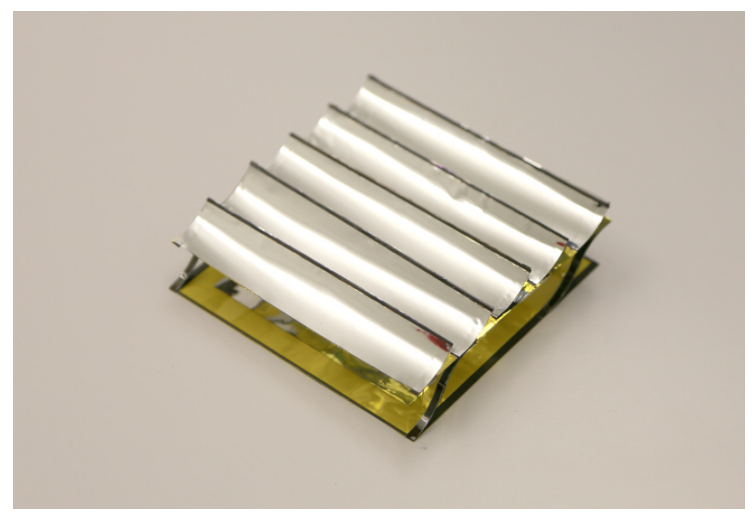

(a)

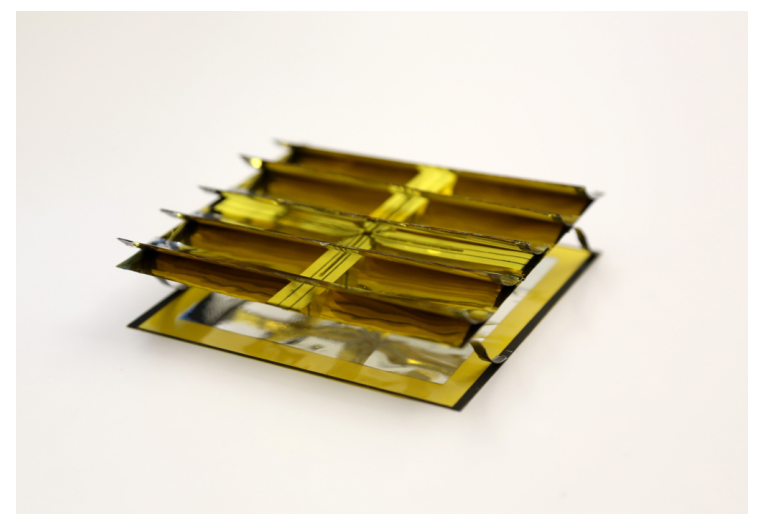

(b)

Figure 3: Tile mockup.

This mockup includes an integrated circuit and five photovoltaic cells. However, these components are simply mass and structural simulators, and not intended for operation.

The initial $10 \mathrm{~cm} \times 10 \mathrm{~cm}$ tile mockup has a mass of $1.56 \mathrm{~g}$. The tile mass is expected to decrease to at least $0.8 \mathrm{~g}$, allowing the overall spacecraft areal density to reach its goal of $100 \mathrm{~g} \mathrm{~m}^{-2}$. 


\section{Structural Architecture}

This section discusses the overall structural architecture for a single spacecraft and describes the structural members that hold the tiles. A simple numerical model is used to arrive at preliminary structural parameters for the various spacecraft structural components.

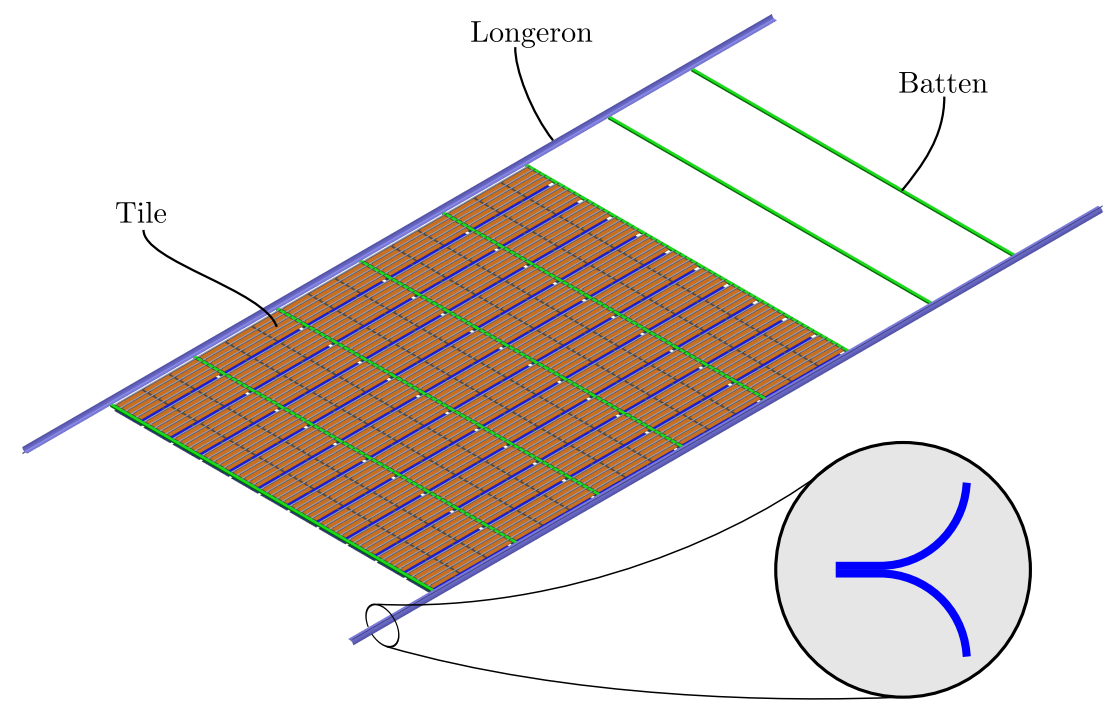

Figure 4: A short segment of a single strip. The longeron has a cross-section similar to a TRAC boom. For clarity, some of the tiles have been omitted.

The tiles are arranged together in strips. All strips have the same width, but may have different lengths. Figure 4 shows a segment of a strip. Two longerons run the entire length of the strip and support its edges. In Figure 4, battens connect the tiles to the longerons.

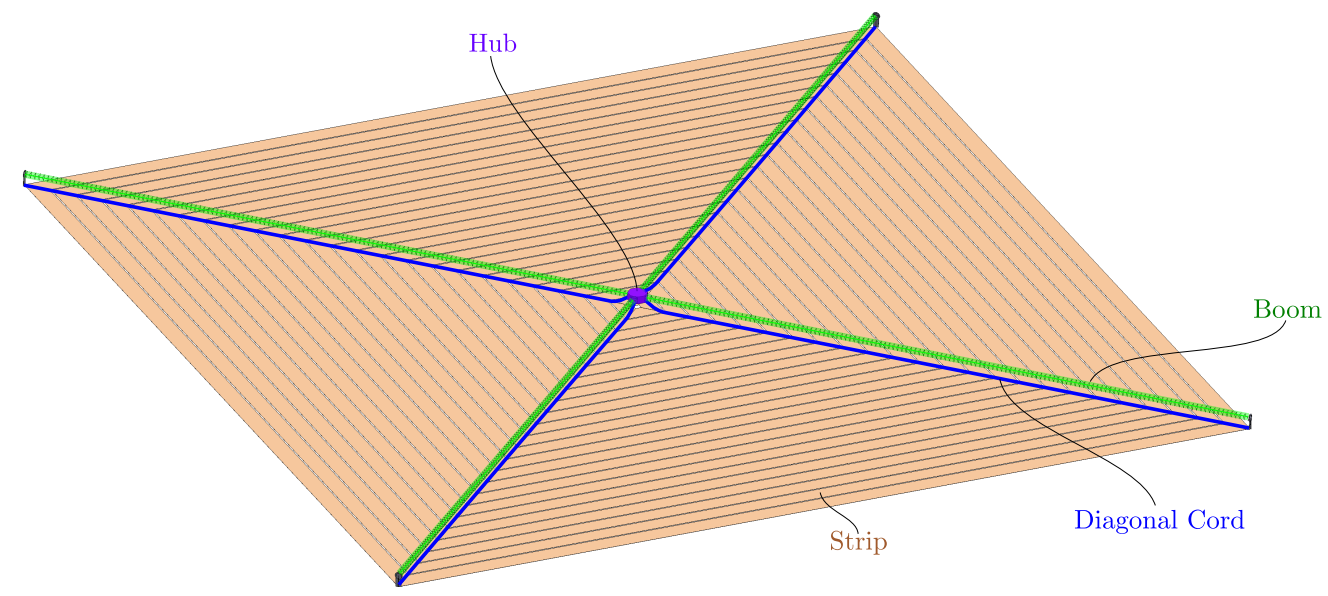

Figure 5: Spacecraft structural architecture.

The strips are arranged in concentric squares, as shown in Figure 5. They are connected at either end to diagonal cords. At one end, the diagonal cords are attached to a central hub, and at the other end, the diagonal cords are connected to tips of deployable booms (e.g. the Northrop Grumman AstroMast ${ }^{16}$ or the ATK coilable booms ${ }^{17}$ ). The booms, clamped to the hub at the center, are located along the diagonals of the squares. These booms provide the motive action during deployment. Each strip is connected to its neighboring strips using ligaments, which allow for the transmission of tension between strips. These ligaments implement the slipping folds crucial to the packaging scheme described in Section V. 
The out-of-plane bending stiffness of the strips is provided primarily by the longerons at the edge of strips. To enable the spacecraft packaging scheme, these longerons must be able to be flattened and rolled. ${ }^{18}$ There are many existing structural elements that would suffice as longerons e.g. STEM booms, ${ }^{19}$ lenticular booms ${ }^{20}$ and TRAC booms. ${ }^{21}$ The current strip design calls for carbon fiber composite TRAC longerons, since the TRAC structure is simpler and has a smaller packaged height.

After deployment, the diagonal cords are tensioned, and the cord tension is reacted by the booms.

\section{IV.A. Numerical Structural Model}

Given this structural architecture, the individual structural elements must be designed. To do so, a simple structural model was created and implemented in MATLAB. It was used to optimize the spacecraft structure, using a loading case and performance metric described below.

The strips were modeled as beams. The ligament connections between the strips were not accounted for in this initial model. The diagonal cords were modeled as lines under tension, and the booms were modeled as beam-columns. For fixed side length $L=60 \mathrm{~m}$, this simplified model has only four structural parameters that control the deflected shape of the spacecraft: the bending stiffness of the boom $E I_{\text {boom }}$, the bending stiffness of a strip $E I_{\text {strip }}$, the number of strips in a quadrant $k$ (which controls the width $w$ of each strip), and the diagonal cord tension $T$.

The spacecraft side length is not taken to be a design parameter; it is fixed at $60 \mathrm{~m}$. This is because the choice of this dimension has effects beyond what can be captured using the present performance metrics.

\section{IV.A.1. Spacecraft Loading}

The loading experienced by the deployed spacecraft can be divided into two classes: dynamic and quasi-static.

Expected sources of dynamic loading are attitude control forces and vibrational noise from attitude control actuators (e.g. thrusters, reaction wheels, or control moment gyros). Assuming that the attitude control system has been designed to decouple structural dynamics from the spacecraft attitude dynamics, the attitude control forces will be small. If the actuators are sufficiently isolated from the structure, the vibrational noise from these sources will be negligible.

Expected sources of quasi-static loading are solar radiation pressure, gravity gradient, and D'Alembert forces produced during attitude control maneuvers. Of these, solar radiation pressure is expected to dominate; the spacecraft will operate in a geosynchronous orbit where gravity gradient forces are minimal, and the attitude control maneuvers are expected to be slow. Therefore solar radiation pressure loading of $B=9 \mu \mathrm{Pa}$ will be the loading case used in the structural design.

For this initial structural design, only quasi-static loading cases will be considered.

\section{IV.A.2. Performance Metric}

A key metric in the design of the spacecraft is the specific power, which is the amount of power delivered to the ground station per unit mass of spacecraft. Since the present exercise deals exclusively with the structural design, the effects of the structural design on the specific power will be isolated and considered independently. In particular, the effects of structural deflections on power generation and transmission are considered, as is the mass of the structural components.

The most efficient tile arrangement is with all tiles pointed directly at the sun (which maximizes power generation efficiency), arrayed regularly in a single plane (which maximizes power transmission efficiency). Any angular deviations from such an arrangement reduce the performance of the concentrators, and any translational deviations reduce the performance of the microwave phased array.

If the translational deviations are small enough and slow enough, they can be measured and corrected for by introducing appropriate phase delays at each tile location. A system to perform these measurements and corrections will need to be implemented. Thus, the present structural design exercise will consider only the effect of angular deviations from the nominal planar configuration of the tiles.

The performance metric used to evaluate the structural design is the specific concentrated power: the total power concentrated on the photovoltaic cells divided by the total mass of the spacecraft. The total concentrated power depends on the incoming solar power flux (taken to be constant at $1370 \mathrm{~W} \mathrm{~m}^{-1}$ ) and the average tile concentrating efficiency. 


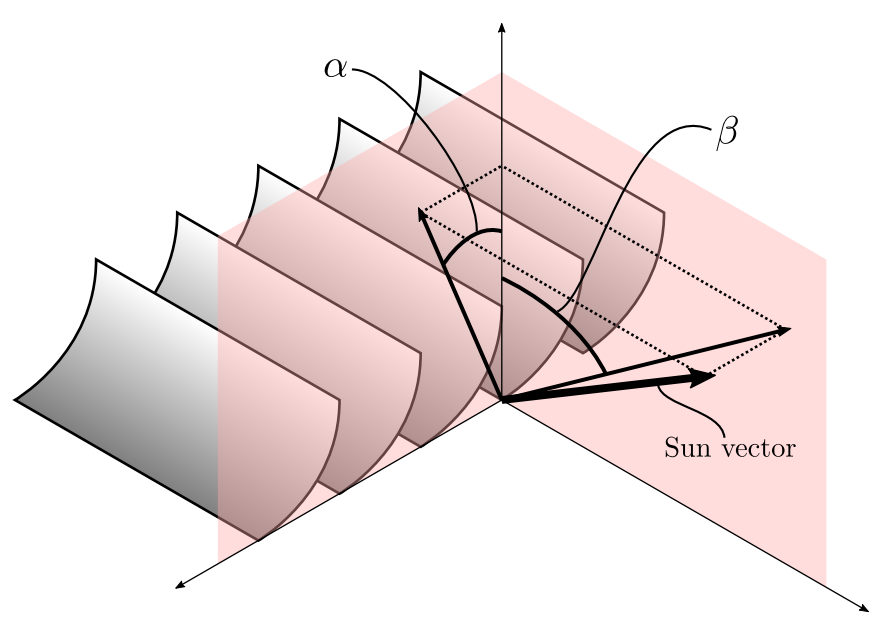

Figure 6: The sun vector at each tile is decomposed into a component in the plane of concentration, at an angle $\alpha$ to the tile normal, and a component perpendicular to the plane of concentration, at an angle $\beta$.

The performance of the concentrators in the tiles depends on the local sun angle. As shown in Figure 6 , the local sun vector can be decomposed into a component within the plane of concentration, and a component perpendicular to this plane. The optical efficiency of the concentrators depends on the $\alpha$ and $\beta$ angles these components make with the local tile normal. As seen in Figure 7, the sensitivity of the concentrating efficiency to $\alpha$ is much greater than the sensitivity to $\beta$.

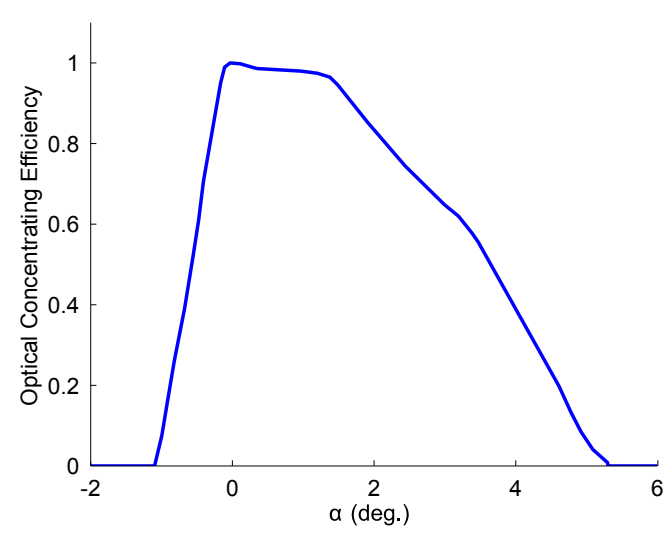

(a)

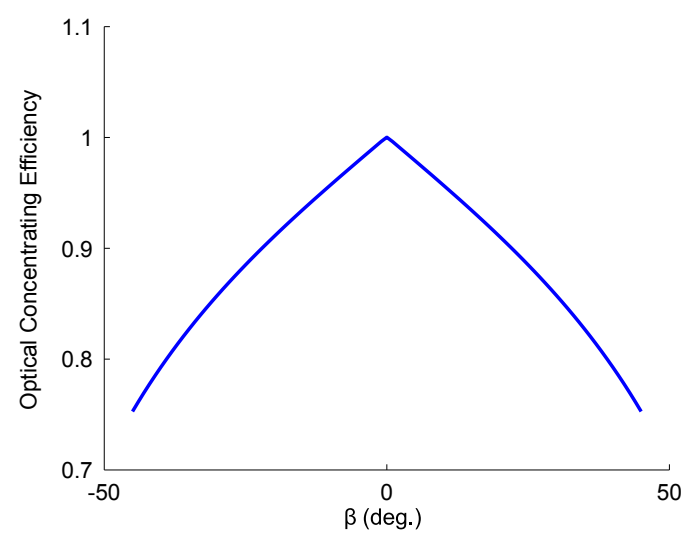

(b)

Figure 7: Tile concentrating efficiency variation with incident sun angles $\alpha$ and $\beta$. These plots were generated by Drs. Pilar Espinet and Dennis Callahan from the research group of Dr. Harry Atwater.

In the present study, the concentrators across the entire spacecraft were arranged to be all parallel. This is because the concentrators are much more sensitive to the $\alpha$ angle than the $\beta$ angle. Thus, the spacecraft can slew in a manner that changes the $\beta$ angle without greatly affecting the concentrating efficiency. If (as an alternative) the tiles were arranged in a 4 -fold symmetric manner, the spacecraft would have to remain very closely sun-pointed (being able to deviate less $1^{\circ}$ in either axis) to generate any power from more than half the tiles. But since the concentrators are all parallel, the spacecraft can slew $\pm 20^{\circ}$ in the $\beta$ direction allowing for operational freedom.

For this initial analysis, it is assumed that the spacecraft is pointed directly at the sun. Due to solar radiation pressure, the structure deflects out-of-plane. To compute these deflections, the following structural model was constructed.

The booms were modeled as beam-columns, with the following relation between the end moments $M_{1}, M_{2}$, 
tip shear load $B L^{2} / 4$, end rotations $\theta_{1}, \theta_{2}$, and tip deflection $u_{\text {boom }}$ :

$$
\left[\begin{array}{c}
M_{1} \\
M_{2} \\
-B L^{2} / 4
\end{array}\right]=\mathbf{K}\left[\begin{array}{c}
\theta_{1} \\
\theta_{2} \\
u_{\text {boom }}
\end{array}\right]
$$

The stiffness matrix $\mathbf{K}$ is a function of the boom bending stiffness $(E I)_{b o o m}$, the boom length $\sqrt{2} L / 2$, and the axial compressive load $T$. It can be found in standard texts. ${ }^{22}$

The booms are clamped at the hub and pinned to the diagonal cords; thus the boundary conditions are $\theta_{1}=0$ and $M_{2}=0$. Using these, Equation (1) can be reduced and inverted to find the tip deflection $u_{\text {boom }}$ :

$$
\left[\begin{array}{c}
\theta_{2} \\
u_{\text {boom }}
\end{array}\right]=\tilde{\mathbf{K}}^{-1}\left[\begin{array}{c}
0 \\
-B L^{2} / 4
\end{array}\right]
$$

The strips were modeled as simply supported beams, loaded normally by a distributed loading $B w$. The vertical deflection of the $i^{\text {th }}$ strip is

$$
u_{\text {strip }, i}(x)=-\frac{B w L_{i}^{4}}{8(E I)_{\text {strip }}}\left[\frac{1}{3}\left(\frac{x}{L_{i}}\right)^{4}-\frac{1}{2}\left(\frac{x}{L_{i}}\right)^{2}\right]+u_{D C, i}
$$

where $x \in\left[-L_{i} / 2, L_{i} / 2\right]$ is a coordinate along the strip, $L_{i}=L i / k$ is the length of the $i^{t h}$ strip, and $u_{D C, i}$ is the vertical deflection at the point at which the strip is attached to the diagonal cord.

The distance between these attachment points is $\sqrt{2} w$. These attachment points are assumed to deflect only vertically; an attachment point cannot deflect in the circumferential direction due to 4 -fold rotational symmetry, and the deflection in the radial direction is assumed to be negligible.

The vertical deflection of the $(i+1)^{t h}$ attachment point on the diagonal cord $u_{D C, i+1}$ can be computed by assuming small vertical deflections, and thus uniform tension $T$ throughout the diagonal cord, hence:

$$
u_{D C, i+1}=2 u_{D C, i}-u_{D C, i-1}-2 \sqrt{2} \frac{B w^{3} i}{T}
$$

The innermost attachment point (i.e. $i=0$ ) is fixed to the hub, thus $u_{D C, 0}=0$. The outermost attachment point is pinned to the tip of the boom, thus $u_{D C, k}=u_{\text {boom }}$. The diagonal cord deflections are computed by a shooting method to satisfy these boundary conditions.

To find the $\alpha$ and $\beta$ angles at a tile on the $i^{\text {th }}$ strip at a location $x$ along the strip, the local tile normal $\mathbf{n}_{t}(x ; i)$ was computed. The local tile normal $\mathbf{n}_{t}(x ; i)$ was obtained by tilting the undeflected normal $[0,0,1]^{T}$ through two rotations: $\mathbf{R}_{1}$ due to the diagonal cord deflections by an angle $\left(u_{D C, i-1}-u_{D C, i}\right) / \sqrt{2} w$, and $\mathbf{R}_{2}$ due to the strip deflections by an angle $u_{\text {strip }, i}^{\prime}(x)$. Thus, at each tile location, the local sun angles $\alpha$ and $\beta$ have non-zero values.

Using the curves shown in Figure 7, the efficiencies due to these angular deformations were found, and the tile concentrating efficiency was computed as the product of these efficiencies. The average tile concentrating efficiency over the entire spacecraft was then evaluated, and multiplied by the spacecraft area and solar flux to compute the total concentrated power.

The other component of the performance metric is the spacecraft mass $m$. It was estimated by accounting for the mass of the tiles $\left(m_{\text {tiles }}\right)$, the hub $\left(m_{\text {hub }}\right)$, the strip structure (excluding the tiles) $\left(m_{\text {strips }}\right)$, the booms $\left(m_{\text {booms }}\right)$, and the diagonal cords $\left(m_{\text {cords }}\right)$.

$$
m=m_{\text {tiles }}+m_{\text {hub }}+m_{\text {strips }}+m_{\text {booms }}+m_{\text {cords }}
$$

The tile mass was calculated by multiplying the expected tile areal density of $80 \mathrm{~g} \mathrm{~m}^{-2}$ by the total spacecraft area. The tile mass does not change with changes in the structural design of the spacecraft:

$$
m_{\text {tiles }}=\sigma_{\text {tiles }} L^{2}=80 \mathrm{~g} \mathrm{~m}^{-2} \times 60 \mathrm{~m} \times 60 \mathrm{~m}=288 \mathrm{~kg}
$$

The hub mass was assumed to be fixed: $m_{h u b}=50 \mathrm{~kg}$. This estimate is based on the use of nanosatellite components and includes the propulsion system. 
The mass of the strip structure was calculated by homogenized strip linear density $\lambda_{\text {strip }}$ (accounting for the cross-sectional area of the longerons, the density of the longeron material, and the battens), and multiplying it by the total strip length:

$$
\begin{aligned}
m_{\text {strips }} & =2 L(k+1) \lambda_{\text {strip }} \\
\lambda_{\text {strip }} & =2 \lambda_{\text {longeron }}+\lambda_{\text {batten }} \frac{L}{2 k p_{\text {batten }}}
\end{aligned}
$$

where $\lambda_{\text {longeron }}$ is the longeron linear density, $\lambda_{\text {batten }}$ is the batten linear density, $p_{\text {batten }}$ is the batten pitch, $L$ is the spacecraft side length, and $k$ is the number of strips per quadrant. The batten linear density $\lambda_{\text {batten }}=0.16 \mathrm{~g} \mathrm{~m}^{-1}$ and the batten pitch $p_{\text {batten }}=30 \mathrm{~cm}$ are fixed. The longeron linear density $\lambda_{\text {longeron }}$ is calculated as the product of the longeron cross-sectional area and the material volumetric density (taken to be $1600 \mathrm{~kg} \mathrm{~m}^{-3}$ ).

The boom mass was estimated by using a homogenized linear density $\left(\lambda_{\text {boom }}\right)$.

The diagonal cord mass was calculated by estimating an appropriate cross-sectional area (taken to be the area that results in $0.1 \%$ strain given the desired diagonal cord pre-tension $T$ ), and using this area to calculate the diagonal cord linear density (using a volumetric density of $1600 \mathrm{~kg} \mathrm{~m}^{-3}$ ).

\section{IV.A.3. Structural Model Results}

For initial modeling efforts, the booms were assumed to have the properties of the ATK Coilable Boom for the ST8 Sailmast with $(E I)_{\text {boom }}=8035 \mathrm{~N} \mathrm{~m}^{2}$ and $\lambda_{\text {boom }}=70 \mathrm{~g} \mathrm{~m}^{-1} \cdot{ }^{17}$

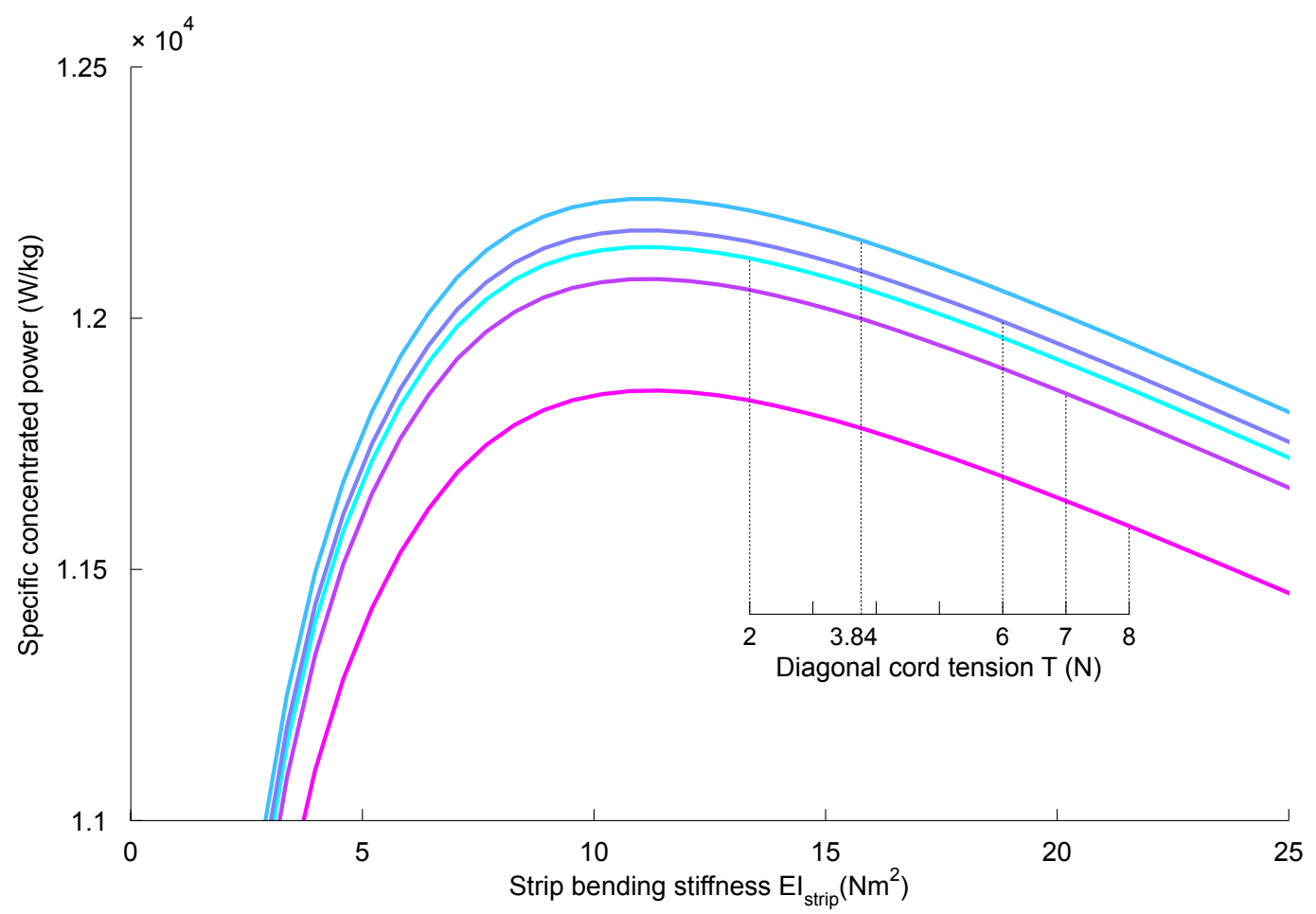

Figure 8: Specific concentrated power as a function of diagonal cord tension $T$ and strip bending stiffness $(E I)_{\text {strip. }}$.

Figure 8 shows how the specific concentrated power changes with the strip bending stiffness and the diagonal cord tension for an optimal value of $k=20$. The optimal design exists at a diagonal cord tension $T=3.84 \mathrm{~N}$ and strip bending stiffness $(E I)_{\text {strip }}=10.78 \mathrm{~N} \mathrm{~m}^{2}$. Increased diagonal cord tension initially stiffens the spacecraft; after a certain point, however, increased compression in the boom reduces its effective bending stiffness, resulting in greater deflections. Increasing the strip bending stiffness also results in initial increases in specific concentrated power, but after a certain point, the mass growth due to larger TRAC boom cross-sections outpaces the growth in collected power from lower deflections. 


\section{Spacecraft Packaging Concept}

The spacecraft packaging concept relies on slipping folds that connect the strips to each other. A slipping fold allows for both rotation about and translation (or slip) along the axis of the fold. Slipping folds allow for membranes and membrane-like structures to be folded and wrapped tightly and efficiently, while accommodating the finite thickness of each strip. Crucially, the strips experience no extension during folding and the maximum bending stresses in the wrapped state can be predicted and controlled. Previous work has demonstrated that for parallel slipping folds, packaging efficiencies of up to $73 \%$ can be achieved. ${ }^{9}$

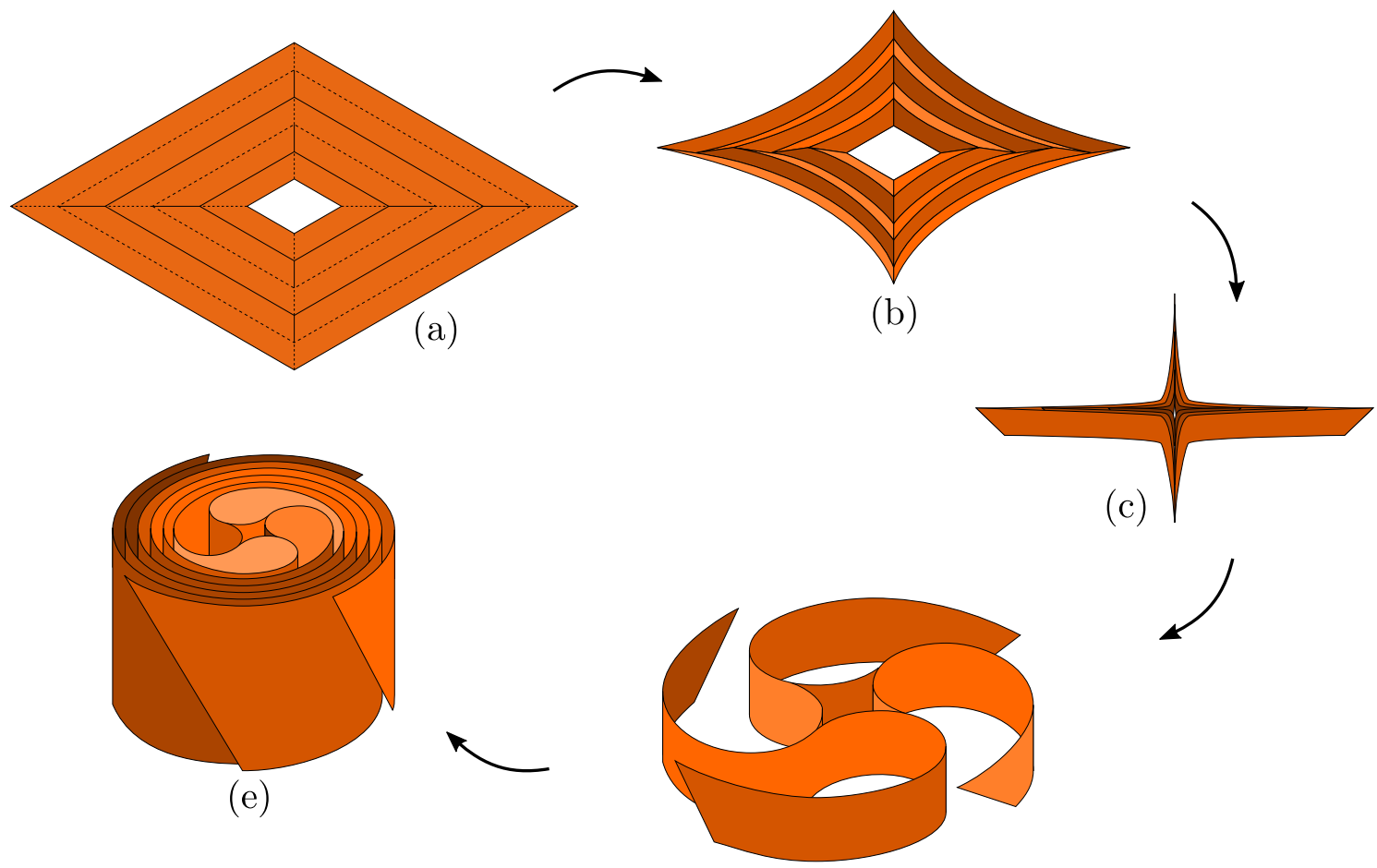

(d)

Figure 9: Spacecraft packaging concept. For clarity, only the outermost strips are shown in (d) and (e)

Consider a fold pattern, as shown in Figure 9a, consisting of $k$ concentric equally spaced squares, alternating between mountain and valley folds. Additional folds run along the diagonals of the squares, creating degree- 4 vertices (points at which 4 folds meet) at every corner of every square (except the innermost and outermost squares). Note that this folding scheme can be generalized for any regular polygon.

Folding along these lines produces a star-like shape with four arms, as shown in Figure 9c. Wrapping these arms results in a compact packaged cylindrical form (see Figure 9e). There are five voids in the packaged form; one in the center, and one associated with each wrapped arm.

Neither the folding nor the subsequent wrapping is novel. The fold pattern itself has been described and studied, ${ }^{23}$ and it was used, along with the wrapping step, to package the IKAROS solar sail. ${ }^{10}$

The key innovative step here is the use of slipping folds to implement this fold pattern. Without slipping folds, this method of packaging does not accommodate the thickness $h$ of the material being folded. Another way to accommodate thickness in this fold pattern is to use curved crease lines, ${ }^{24,25}$ but curved creases are not favorable, since they would disrupt the regular placement of tiles. Slipping folds allow for adjacent strips to slide past each other, accounting for the different radii of the strips in the wrapped configuration.

Figure 10 shows the folding concept demonstrated on a $1 \mathrm{~m} \times 1 \mathrm{~m}, 50 \mu \mathrm{m}$-thick Mylar membrane with 11 strips per quadrant. Packaged, it occupies a cylinder of $51 \mathrm{~mm}$ diameter and $40 \mathrm{~mm}$ height. This membrane model was constructed by laser-cutting slits in a Mylar film. The slits are interrupted by continuous ligaments between the strips. These ligaments are $1 \mathrm{~mm}$ wide and allow for slip between the strips, but can still transfer tension between strips. Similar structures will be used in the full-scale spacecraft to connect the strips. 

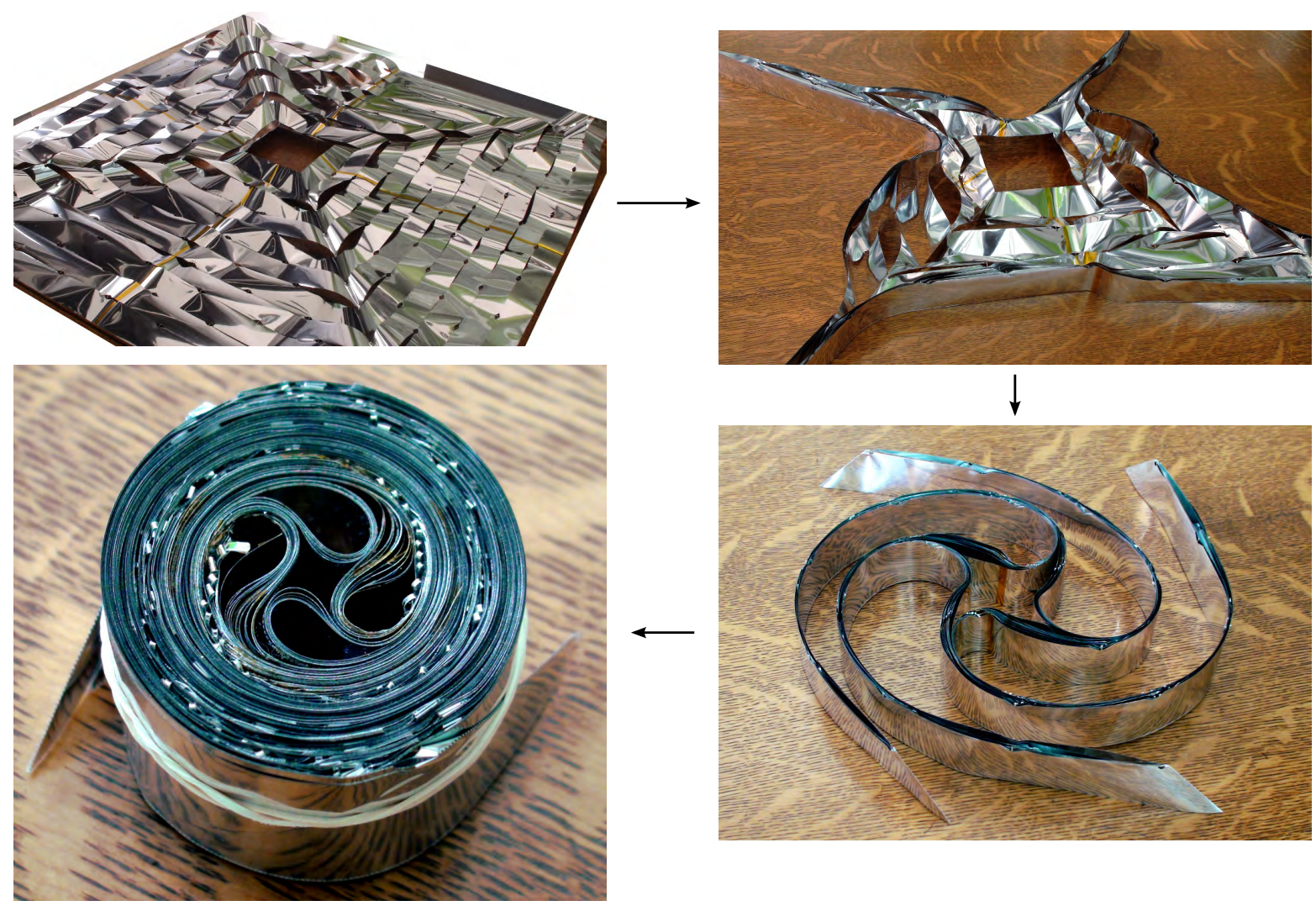

Figure 10: Spacecraft packaging concept demonstrated using a $1 \mathrm{~m} \times 1 \mathrm{~m}, 50 \mu \mathrm{m}$-thick Mylar membrane. Packaged, it occupies a cylinder of $51 \mathrm{~mm}$ diameter and $40 \mathrm{~mm}$ height.

\section{V.A. Kinematic Model of Wrapping}

In this subsection, key descriptors of the packaging scheme will be estimated: the packaged height and the packaged diameter, the packaging efficiency, and the maximum slip.

Consider the configuration of the structure after folding but before wrapping, as depicted in Figure 9c. Taking a planar slice through this configuration, as shown in Figure 11a, produces a set of 2D curves. These curves trace the location of every strip after folding. Similar curves that trace the strips after wrapping can be used to describe the fully packaged structure.

Note how the strips in a single quadrant have different lengths. This leads to an arm with variable thickness, and wrapping this variable-thickness arm produces a spiral-like shape where the pitch of the spiral decreases as one moves outwards. Our previous work ${ }^{9}$ modeled the wrapping of a stack of strips with uniform thickness using an involute of a circle: a spiral curve that maintains constant spacing between successive turns. To use a similar method here, assume that the the strips have equal length $L$, as shown in Figure 11b. This leads to arms that have uniform thickness $2 k h$, and, in the wrapped state, can be described by an involute of a circle.

The assumption of equal strip length is unphysical and incompatible with the folding pattern. However, it simplifies the modeling of the wrapped form and allows for the use of existing techniques. More importantly, since this assumption accounts for more material than is physical, it provides a conservative upper bound on the packaged diameter, packaging efficiency, and maximum slip.

With this assumption, only a single $2 \mathrm{D}$ curve, called the base curve $\mathbf{r}(s)$ need be described, as shown in Figure 12. All strips in a single quadrant follow curves that are parallel to this base curve, and the strips in the other quadrants are related through 4 -fold rotational symmetry. The base curve is shown as the thick curve in Figure 12. It consists of three parts: the dotted curve $\mathbf{q}(s)$, the solid curve $\mathbf{p}(s)$, and a dashed 


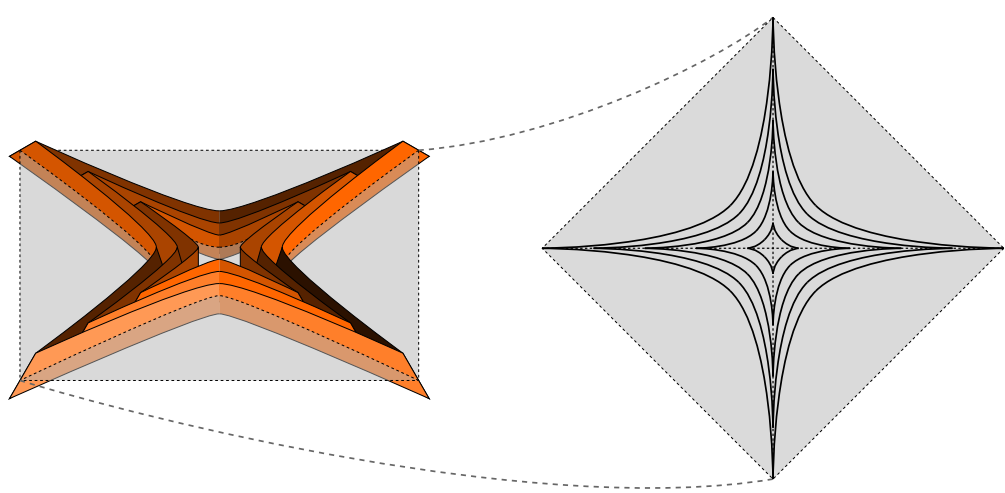

(a)

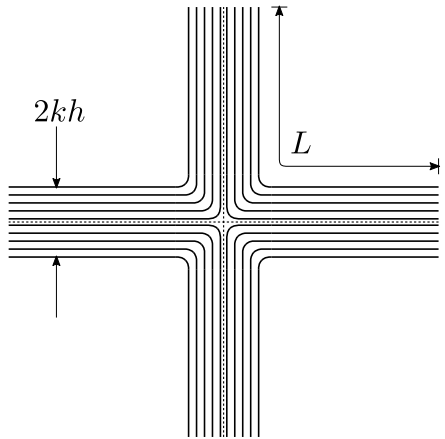

(b)

Figure 11: (a) The curves that trace the paths of the strips can be generated by taking a slice through the folded form. (b) Equal strip length $L$ is assumed to produce arms of constant thickness $2 k h$.

curve, which is copy of $\mathbf{p}(s)$ rotated clockwise $90^{\circ}$.

$$
\mathbf{r}(s)= \begin{cases}\mathbf{R} \mathbf{p}(-s) & \text { if } s \in\left[-L / 2,-\left(L / 2+s_{p}\right)\right) \\ \mathbf{q}(s) & \text { if } s \in\left[-\left(L / 2+s_{p}\right),\left(L / 2+s_{p}\right)\right) \\ \mathbf{p}(s) & \text { if } s \in\left[\left(L / 2+s_{p}\right), L / 2\right]\end{cases}
$$

where $\mathbf{R}$ is a rotation matrix for the clockwise $90^{\circ}$ rotation, and $s_{p}$ is the arclength of the curve $\mathbf{p}$.

The $i^{t h}$ strip follows a curve $\mathbf{r}(i ; s)$ that is parallel to the base curve, separated by a multiple of the thickness $h$ :

$$
\mathbf{r}(i ; s)=\mathbf{r}(s)+\left(i+\frac{1}{2}\right) h \mathbf{n}(s)
$$

where $\mathbf{n}(s)$ is the normal to the base curve.

$\mathbf{p}(s)$ and $\mathbf{q}(s)$ are constructed in a piecewise manner. Their components are shown in Figure 13. $\mathbf{q}(s)$ is a quarter-circle $A B$ of radius $R_{0}$ centered at a point $\mathbf{O}_{0} . \mathbf{p}(s)$ consists of four pieces:

1. $B C$, which is a continuation of circle $A B$

2. $C D$, which is a circle of radius $R_{1}$, centered at $\mathbf{O}_{1}$, and tangent to $B C$ at $C$

3. DE, which is a vertical line segment, and

4. EF, which is an involute of a circle.

The spacing between the arms of the involute is $2 \pi f=4 \times 2 k h$ where $2 k h$ is the thickness of each arm. The factor of 4 accounts for the four arms being wrapped around.

The radius of small circle $R_{0}=R_{\min }+(k+1 / 2) h$ is such that the curvature limit $1 / R_{\text {min }}$, dictated by some maximum uniaxial strain limit $\epsilon_{\max }$, is not exceeded for the outermost strip

$$
R_{\min } \geq \frac{h}{2 \epsilon_{\max }}
$$

Note that this curve has discontinuous curvature at all points where two pieces meet. It is not expected that a wrapped membrane will follow this curve exactly; however, it is a simple curve that may be used to estimate key parameters. 


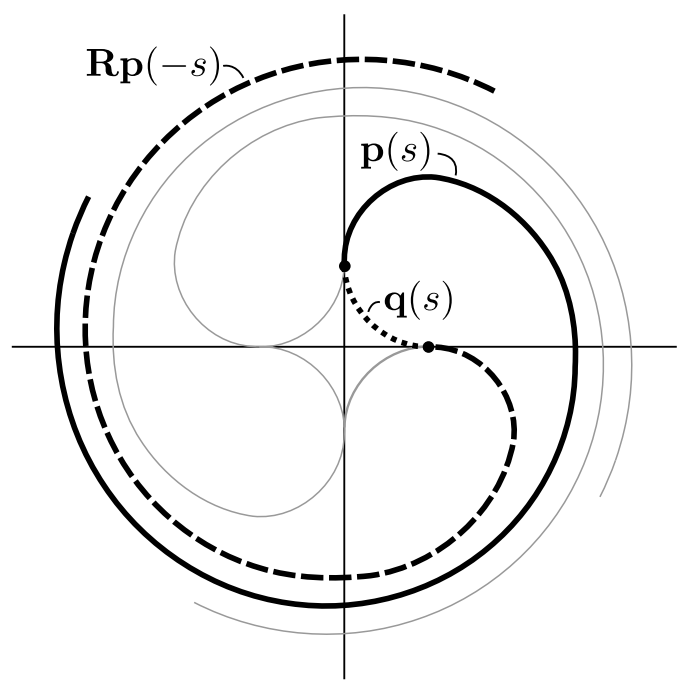

(a)

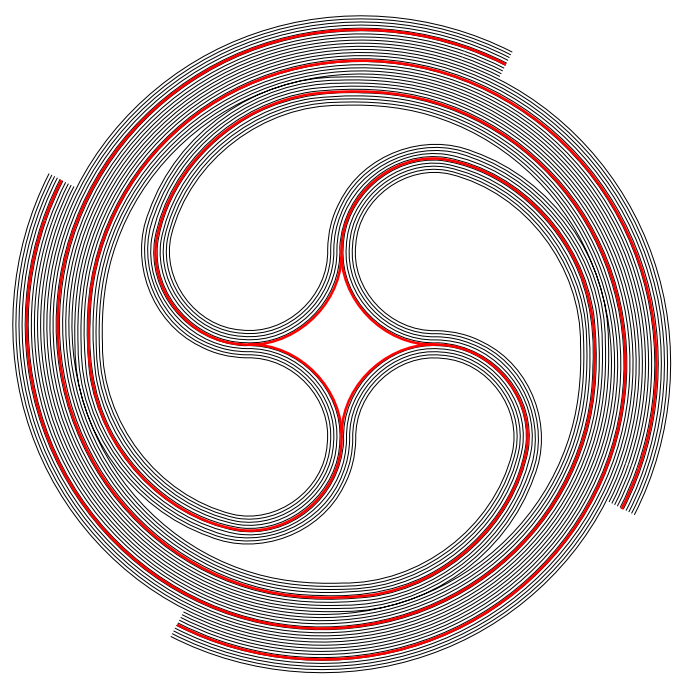

(b)

Figure 12: (a) Base curve $\mathbf{r}$ used to model the wrapped strips, which consists of three generator curves: $\mathbf{R} \mathbf{p}(-s), \mathbf{q}(s)$, and $\mathbf{p}(s)$. (b) The strips follow curves parallel to the base curve.

\section{V.B. Packaging Efficiency}

The packaging efficiency $\eta$ is defined as the ratio of the material volume of the structure $V_{m}$ to the volume of the container $V_{p}$ into which it is packaged. Based on the assumption of equal strip length, the material volume is taken to be the volume of the $4 k$ strips of length $L$, width $w$, and thickness $h$. The container that holds the packaged structure is taken to be a cylinder of height $H_{p}$ and radius $R_{p}$. Now the cylinder height $H_{p}$ is exactly the strip width $w$, which gives the following expression for $\eta$ :

$$
\eta=\frac{4 k L h}{\pi R_{p}^{2}}
$$

The radius of the cylinder $R_{p}$ is the radius of the outermost point on any strip:

$$
R_{p}=\max _{i, s}\|\mathbf{r}(i ; s)\|
$$

The packaging efficiency $\eta$ can be calculated to be

$$
\begin{aligned}
\eta & =\frac{4 \lambda}{\pi k}\left[\left(\frac{4}{\pi}\right)^{2}+\left(\frac{4 \alpha_{\max }}{\pi}\right)^{2}+\frac{8 \alpha_{\max }}{\pi}+1\right]^{-1} \\
\alpha_{\max }^{2} & =\frac{\pi s_{v}}{2 k h}+\left(\frac{\pi a}{4 k h}\right)^{2}
\end{aligned}
$$

where $\lambda$ is the ratio of the structure side length $L$ to the flattened strip thickness $h, s_{v}$ is the arclength of the circle involute, and $a$ is a geometrical parameter equal to the $x$ co-ordinate of point $D$ in Figure 13. Figure 14 plots the packaging efficiency as a function of $\lambda$, for a variety of values of $R_{\min } / h$.

\section{V.C. Maximum Slip}

The slip $l(i ; s)$ between the $(i+1)^{t h}$ and the $i^{t h}$ strip in a quadrant is defined in previous work ${ }^{9}$ as the difference between the arc lengths $s_{i+1}$ and $s_{i}$ of the two curves. The arc lengths $s_{i}$ are parameterized by the arc length $s$ of the base curve. The slip between two strips has the expression: ${ }^{9}$

$$
l(s)=h \int_{s_{0}}^{s} \kappa(\xi) \mathrm{d} \xi
$$




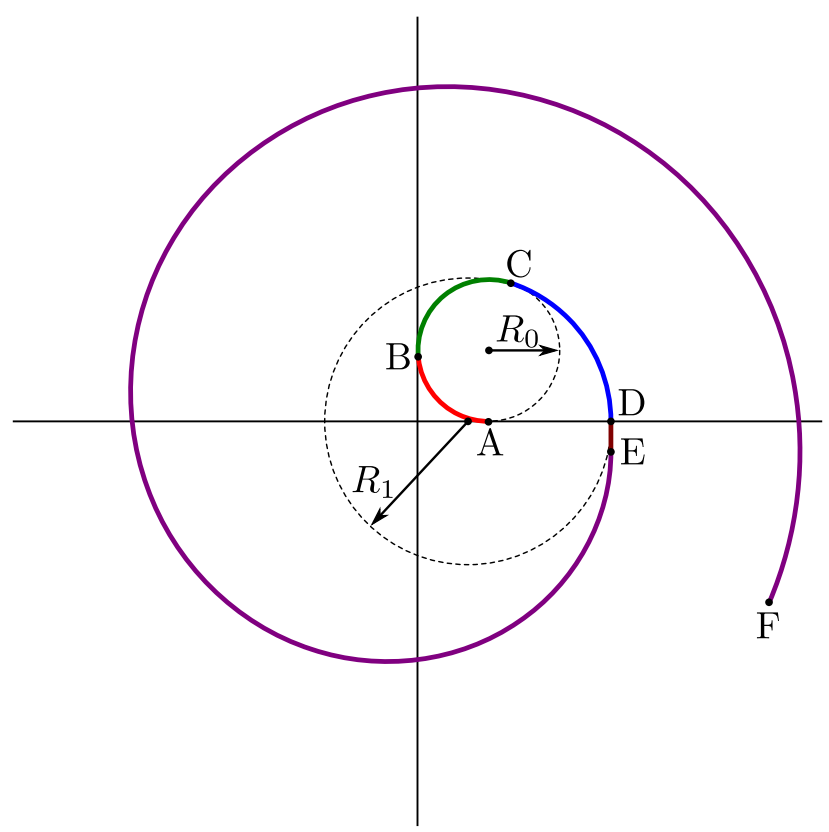

Figure 13: Components of the generator curves $\mathbf{q}$ and $\mathbf{p}$. $\mathbf{q}$ consists of the curve $A B$, and $\mathbf{p}$ consists of the curve $B C D E F$.

where $\kappa(s)$ is the signed curvature of the base curve, and $s_{0}$ is some location where the slip is defined to be identically zero. Notably, the slip is independent of the strip index $i$.

The maximum slip occurs at the middle of the base curve:

$$
\begin{aligned}
l_{\max } & =h \int_{-L / 2}^{0} \kappa(\xi) \mathrm{d} \xi \\
& =h\left(\alpha_{\max }-\frac{\pi a}{4 k h}+\frac{5 \pi}{4}\right)
\end{aligned}
$$

\section{Spacecraft Design Summary}

This section collects relevant results from previous sections to present a preliminary design of a single spacecraft.

From Section IV.A.3, the optimal structural design is found to exist at $k=20$ (i.e. 20 strips of $1.5 \mathrm{~m}$ width in a single quadrant), diagonal cord tension $T=3.84 \mathrm{~N}$ and strip bending stiffness $(E I)_{\text {strip }}=10.78 \mathrm{~N} \mathrm{~m}$. The mass of this optimal $60 \mathrm{~m} \times 60 \mathrm{~m}$ structure is found to be $368.89 \mathrm{~kg}$, leading to an overall areal density of $102.47 \mathrm{~g} \mathrm{~m}^{-2}$. Table 1 lists the mass of the various spacecraft components.

\begin{tabular}{lr} 
Component & Mass $(\mathbf{k g})$ \\
\hline Tiles & 288.00 \\
Strip structure & 19.00 \\
Hub & 50.00 \\
Booms & 11.88 \\
Diagonal cords & 0.01 \\
\hline Total & 368.89
\end{tabular}

Table 1: Spacecraft mass breakdown.

To achieve the desired strip bending stiffness of $10.78 \mathrm{~N} \mathrm{~m}$, the two longerons supporting the strip must each have a bending stiffness of half this value, i.e. $5.39 \mathrm{~N} \mathrm{~m}$. Assuming a Young's modulus of $140 \mathrm{GPa}$ (typical 


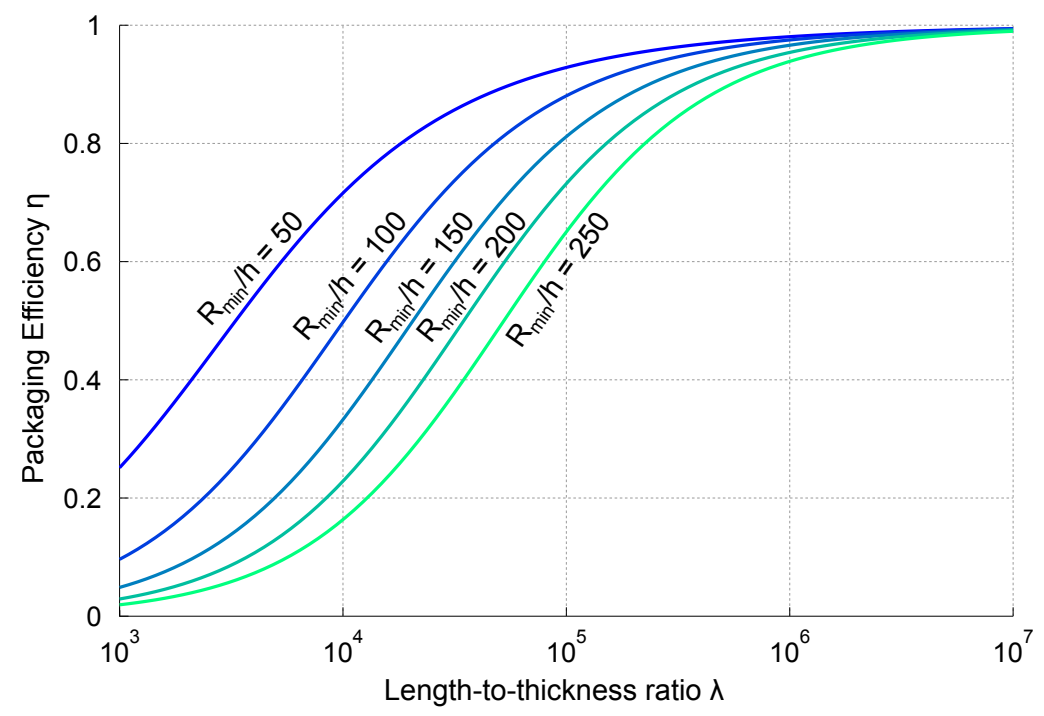

Figure 14: Packaging efficiency $\eta$ as a function of length-to-thickness ratio $\lambda=L / h$ and nondimensional minimum radius of curvature $R_{\min } / h$. The number of strips in a quadrant $k$ is held constant at 20 .

of carbon fiber composites), a TRAC cross-section with a flange radius of $10 \mathrm{~mm}$ and a flange thickness of $68.5 \mathrm{um}$ was designed to provide this bending stiffness.

A TRAC flange thickness of $68.5 \mu \mathrm{m}$ leads to a flattened longeron thickness of $137 \mu \mathrm{m}$. Assuming the flattened tiles and the flattened battens are thinner than the flattened longerons, the flattened strip thickness can be taken to be $h=137 \mu \mathrm{m}$. Since $L=60 \mathrm{~m}$, the length-to-thickness ratio is $\lambda=10^{5.64}$.

The minimum radius $R_{\min }$ can be calculated using Equation (11). The longerons are assumed to have a uniaxial strain limit of $1 \%$, with an additional factor of safety of 2 against material failure, thus $\epsilon_{\max }=0.5 \%$.

$$
\frac{R_{\min }}{h}=\frac{1}{2 \epsilon_{\max }}=100 \Rightarrow R_{\min }=13.7 \mathrm{~mm}
$$

From Figure 14, the packaging efficiency of a structure with $\lambda=10^{5.64}$ and $R_{\min } / h=100$ is $95.6 \%$. The packaged dimensions of the spacecraft can be estimated using the kinematic model: a cylinder with diameter of $0.46 \mathrm{~m}$ and a height of $1.50 \mathrm{~m}$. In this packaged form, the maximum slip can be estimated using Equation (18) to be $l_{\max }=16.8 \mathrm{~mm}$. This informs the design of the longerons, as they must be able to provide this degree of slip.

The final result is an ultralightweight spacecraft that measures $60 \mathrm{~m} \times 60 \mathrm{~m}$, has a mass of $369 \mathrm{~kg}$ (of which $288 \mathrm{~kg}$, or $78 \%$, is the tile mass), and can package into a compact cylindrical form. Note that this packaged volume excludes the packaged booms, the hub, and whatever containment and deployment mechanisms that will be required.

\section{Conclusion}

This paper has presented a preliminary structural design of a spacecraft that measures $60 \mathrm{~m} \times 60 \mathrm{~m}$ and that carries many modular multifunctional tiles. A formation of these spacecraft is envisioned to capture solar power in space, and transmit it to a ground station on the Earth.

A design concept for these multifunctional $10 \mathrm{~cm} \times 10 \mathrm{~cm}$ tiles, each of which is capable of power generation and transmission, was described. These tiles are expected to be very lightweight; an initial mockup with a mass of $1.56 \mathrm{~g}$ was constructed, and this mass is expected to decrease by a factor of about 2 .

A novel structural framework was designed, using solar radiation pressure as a loading case and specific concentrated power as a performance metric, to hold these tiles in a planar configuration. Both the framework and the tiles are capable of elastically flattening to a thickness of no more than $137 \mu \mathrm{m}$, to enable the spacecraft to package.

Indeed, the complete structural architecture of this spacecraft was selected to be compatible with the 
packaging technique, which consists of first folding and then wrapping the flattened tiles and framework into a tight cylindrical form. Key to the packaging process are slipping folds - mechanisms that allow both rotation and translation along the fold line. This packaging process is inextensional, and the maximum bending strains in the packaged state can be predicted and controlled. A kinematic model of this packaging process was described and used to estimate the packaged dimensions and maximum slip.

There are many aspects of the structural design of this spacecraft that need refinement and will be addressed in future studies.

Of particular importance is the question of spacecraft deployment; the motions, the stresses, and the forces produced by the structure during unfolding must be studied, modeled, and experimentally tested. Mechanisms to control the deployment process must be designed.

Additionally, the structural response of the spacecraft must be captured with higher fidelity models for additional loading cases, including dynamic excitations.

The mechanical interfaces between the various components of this structure must specified. For instance, the ligaments that implement the slipping folds, the connections between the strips and the diagonal cords, and attachment of the the tiles to the strip structure must be designed.

Given these concerns, the preliminary nature of the spacecraft structural design presented herein must be emphasized. However, this novel structural architecture, if successfully implemented, promises extreme advances in spacecraft size, areal density, and packaging compactness.

\section{Acknowledgments}

Financial support from Northrop Grumman Corporation is gratefully acknowledged. The research presented in this paper was carried out in collaboration with Professors Harry Atwater and Ali Hajimiri and their research groups. Drs. Dennis Callahan and Florian Bohn helped with the design and the construction of the tile mockup.

\section{References}

${ }^{1}$ Glaser, P. E., "Power from the Sun: Its future," Science, Vol. 162, No. 3856, 1968, pp. 857-61.

${ }^{2}$ US Department of Energy and National Aeronautics and Space Administration, "Satellite Power System: Concept Development \& Evaluation Program," Tech. Rep. October, 1978.

${ }^{3}$ Carrington, C., Fikes, J., Gerry, M., Perkinson, D., Feingold, H., and Olds, J., "The Abacus/Reflector and integrated symmetrical concentrator - Concepts for space solar power collection and transmission," 35th Intersociety Energy Conversion Engineering Conference, 2000.

${ }^{4}$ Mankins, J. C., "A Technical Overview of the Suntower Solar Power Satellite Concept," Acta Astronautica, Vol. 50, No. 6, 2002, pp. 369-377.

${ }^{5}$ Mankins, J. C., The Case for Space Solar Power, The Virgina Edition, 2014.

${ }^{6}$ Oda, M., "Realization of the Solar Power Satellite Using the Formation Flying Solar Reflector," NASA Formation Flying Symposium, 2004.

${ }^{7}$ Seboldt, W., Klimke, M., Leipold, M., and Hanowski, N., "European Sail Tower SPS concept," Acta Astronautica, Vol. 48, No. 5-12, 2001, pp. 785-792.

${ }^{8}$ Sasaki, S., Tanaka, K., Higuchi, K., Okuizumi, N., Kawasaki, S., Shinohara, N., Senda, K., and Ishimura, K., "A new concept of solar power satellite: Tethered-SPS," Acta Astronautica, Vol. 60, No. 3, 2007, pp. 53-165.

${ }^{9}$ Arya, M., Lee, N., and Pellegrino, S., "Wrapping Thick Membranes with Slipping Folds," 2nd Spacecraft Structures Conference, 2015.

${ }^{10}$ Furuya, H., Mori, O., Okuizumi, N., Shirasawa, Y., Natori, M. C., Miyazaki, Y., and Matunaga, S., "Manufacturing and Folding of Solar Sail IKAROS," 52nd AIAA/ASME/ASCE/AHS/ASC Structures, Structural Dynamics, and Materials Conference, April 2011, pp. 4-7.

${ }^{11}$ Murphy, D. M., "The Scarlet Solar Array: Technology Validation and Flight Results," Proceedings of the Deep Space I Technology Validation Symposium, 2000.

${ }^{12}$ Wachholz, J. J. and Murphy, D. M., "SCARLET I: Mechanization Solutions for Deployable Concentrator Optics Integrated with Rigid Array Technology," 30th Aerospace Mechanisms Symposium, 1996.

${ }^{13}$ Pappa, R. S., Woods-Vedeler, J. A., and Jones, T. W., "In-Space Structural Validation Plan for a Stretched-Lens Solar Array Flight Experiment," 20th International Modal Analysis Conference, 2002.

${ }^{14}$ O'Neill, M., McDanal, A., Piszczor, M., Eskenazi, M., Jones, P., Carrington, C., Edwards, D., and Brandhorst, H., "The stretched lens ultralight concentrator array," Photovoltaic Specialists Conference, 2000. Conference Record of the TwentyEighth IEEE, 2000, pp. 1135-1138.

${ }^{15}$ Steering Committee for NASA Technology Roadmaps, NASA Space Technology Roadmaps and Priorities: Restoring NASA's Technological Edge and Paving the Way for a New Era in Space, 2012.

${ }^{16}$ Finley, L. A., "Large-diameter Astromast development," 1984. 
${ }^{17}$ Murphy, D. M., McEachen, M. E., Macy, B. D., and Gaspar, J. L., "Demonstration of a 20-m Solar Sail System," 46th AIAA/ASME/ASCE/AHS/ASC Structures, Structural Dynamics \& Materials Conference, 2005.

${ }^{18}$ Tan, L. T. and Pellegrino, S., "Thin-shell deployable reflectors with collapsible stiffeners: Part 1 Approach," AIAA Journal, Vol. 44, No. 11, 2006, pp. 2515-2523.

${ }^{19}$ Rimrott, F., "Storable Tubular Extendable Member: A Unique Machine Element," Journal of Machine Design, Vol. 37, 1965 , pp. 156-165.

${ }^{20}$ Herbeck, L., Eiden, M., Leipold, M., Sickinger, C., and Unckenbold, W., "Development and test of deployable ultralightweight CFRP booms for a Solar Sail," Proceedings of the European Conference on Spacecraft Structures, Materials and Mechanical Testing, 2000, pp. 107-112.

${ }^{21}$ Banik, J. A. and Murphey, T. W., "Performance Validation of the Triangular Rollable and Collapsible Mast," 24th Annual AIAA/USU Conference on Small Satellites, 2010.

${ }^{22}$ Bazant, Z. P. and Cedolin, L., Stability of Structures, World Scientific Publishing Company, 2010.

${ }^{23}$ Demaine, E. D., Demaine, M. L., Hart, V., Price, G. N., and Tachi, T., "(Non)existence of Pleated Folds: How Paper Folds Between Creases," Graphics and Combinatorics, Vol. 27, No. 3, 2012, pp. 377-397.

${ }^{24}$ Satou, Y. and Furuya, H., "Local Buckling in Crease Induced by Wrapping Fold of Space Membrane," 53rd AIAA/ASME/ASCE/AHS/ASC Structures, Structural Dynamics, and Materials Conference, April 2012.

${ }^{25}$ Satou, Y. and Furuya, H., "Fold Line Based on Mechanical Properties of Crease in Wrapping Fold Membrane," $54 t h$ AIAA/ASME/ASCE/AHS/ASC Structures, Structural Dynamics, and Materials Conference, April 2013. 